\title{
湖北渔塘坝硒矿床中最大硒同位素分馏的发现 及其指示意义
}

\author{
温汉捷 ${ }^{\circledR}$ Jean Carignan ${ }^{\ominus}$ 胡瑞忠 ${ }^{\circledR}$ 樊海峰 ${ }^{\circledR}$ 畅 斌 $^{\oplus}$ 杨光树 ${ }^{\circledR}$ \\ (1) 中国科学院地球化学研究所矿床地球化学国家重点实验室, 贵阳, 550002; (2) CRPG-CNRS, 15, Rue Notre-Dame des Pauvres, B. P. \\ 20, 54501, Vandoeuvre-lès-Nancy Cedex, France. E-mail: wenhanjie@vip.gyig.ac.cn)
}

摘要 对渔塘坝硒矿床中高硒的碳质硅质岩和碳质页岩样品进行了硒同位素测定. 测定结果显示, 其 $\delta^{82 / 76} \mathrm{Se}_{\mathrm{NIST}}$ 范围从-12.77\% $\sim 4.93 \%$, 总变化为 $17.7 \%$. 这是迄今所发现的自然界中最大的同位素分馏, 同时样品 Ytb-5(高硒碳质页岩)的 $\delta^{82 / 76} \mathrm{Se}_{\mathrm{NIST}}$ 为 $-12.77 \%$, 也是目前所发现的自然界中最富硒轻同位素 的样品. 根据硒同位素的分布特征, 结合其他地质证据和地球化学指标, 认为“氧化-还原模式”是对矿 床中自然硒大量出现的合理解释. 同时, 硒同位素在自然界中较大的分馏效应也证明其作为一种新的 地球化学示踪剂有其独特的应用潜力.

关键词 硒同位素 同位素分馏 黑色岩系 自然硒 渔塘坝

位于湖北恩施的渔塘坝硒矿床是世界上目前发 现的唯一的以黑色岩系为容矿围岩的独立硒矿床. 硒矿床的赋矿层位严格受下二叠统茅口组含碳硅质 岩与含碳质页岩控制, 共圈定 9 个矿体, 硒的平均品 位为 $0.13 \%$. 这其中, 硒的赋存状态一直是研究者关 注的焦点问题, 不仅因为它是了解硒的来源、运移和 富集的重要地球化学指标, 也是开发和利用硒资源 的关键. 朱建明等人 ${ }^{[1]}$ 于 2000 年在废弃的“石煤”堆 中发现了自然硒晶体, 继而在未风化的含碳硅质岩 中和风化露头中相继发现了自然硒的存在, 由此把 自然硒划分为 3 类: (i ) 原生成因, 指成岩时形成的 自然硒, 后期未发生变化, 此类成因形成的自然硒极 少; (ii ) 次生成因, 指主要指在风化的碳质硅质岩中 由于表生作用形成的自然硒; (iii) “石煤”(高碳页岩) 自燃成因, 主要是人为活动的结果. 2003 年, 我们利 用含碳硅质岩和页岩抽提的干酪根, 通过TEM(透射 电子显微镜)观察发现了硒主要以超微包体硒(纳米 级)的形式黏附或包裹在干酪根中, 由此证明了自然 硒的分布是广泛的 ${ }^{[2]}$. 按照干酪根和原岩的比例以及 干酪根中硒的含量, 我们推测大约 66\%的硒以自然 硒的形式赋存在未风化硅质岩和页岩中, 其余以硫 化物类质同象和硒矿物的形式存在. 然而, 关于硒的 富集机制，特别是其中自然硒的形成机制仍存在重 要分歧, 目前有 3 个观点: (i ) “氧化-还原模式”, 其
模式类似于淋积矿床, 指原岩或贫矿体经化学风化 作用，原先赋存在岩石或矿石中的硒被水带到风化 壳下部的潜水面附近沉淀下来形成矿床 ${ }^{[3,4]}$; ( ii ) 沉 积成因，指成岩过程中同时伴有硒的沉淀，姚林波等

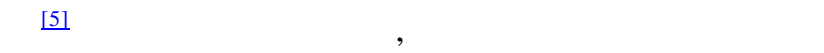
机或微生物还原为自然硒或与硫化物类质同象而沉 积成矿, 在后期没有明显的赋存状态的改变; (iii) 朱 建明等人 ${ }^{[6]}$ 认为部分自然硒是岩层中的“石煤”(高碳 的黑色页岩)自燃或由于构造活动引起的自然硒的出 融引起的. 基于此, 我们试图用国际上新兴的硒同位 素来探讨其可能的富集机制.

样品采集自渔塘坝硒矿床一号矿段. 样品去除 风化表面, 破碎后研磨至 200 目待用. 样品的前处理 采用TCF技术(TCF一Thiol cotton fiber技术，硫醇棉 纤维吸附分离技术), 详细的实验过程和方法详见文 献[7]. 硒同位素测试在法国岩石学地球化学研究中 心(CNRS-CRPG)的Isoprobe MC-ICP-MS(Isoprobe型 多接收器电感耦合等离子质谱)上进行. 部分重复样 在法国里昂的ENS-Lyon实验室的Nu MC-ICP-MS上 测定. 进样系统采用在线氢化物发生器气体进样技 术 (online hydride generator). 用 “Sample-Standard Bracketing”方式校正质量歧视，测试精度达到 $\pm 0.2 \%$ 。 由于目前没有认可的硒同位素国际标样, 我们用新 标定的NIST SRM 3149, Merck, CRPG作为内部同位 
素标准. 以NIST SRM3149 作为同位素参照标准, 其 $\delta$ 定义为 ${ }^{[8]}: \quad \delta^{82 / 76} \mathrm{Se}(\%)=\left[\left(^{82} \mathrm{Se} /{ }^{76} \mathrm{Se}\right){ }_{\text {sample }} /\left({ }^{82} \mathrm{Se} /{ }^{76} \mathrm{Se}\right)_{\text {std }}{ }^{-}\right.$ $1] \times 1000$.

测试结果见表 1. 硒同位素显示了一个较大的分 馏, 其 $\delta^{82 / 76} \mathrm{Se}_{\mathrm{NIST}}$ 范围从-12.77\% $\sim 4.93 \%$, 总变化 为 $17.7 \%$. 这是迄今所发现的自然界中最大的同位素 分馏, 同时样品Ytb-5 的 $\delta^{82 / 76} \mathrm{Se}_{\mathrm{NIST}}$ 为 $-12.77 \%$, 这也 是目前所发现的自然界中最富硒轻同位素的样品. 图 1 列出了目前所收集到的自然样品的 $\delta^{82 / 76} \mathrm{Se}_{\mathrm{NIST}}$ 值 作为对比. 陨石和各种火成岩的 $\delta^{82 / 76} \mathrm{Se}_{\mathrm{NIST}}$ 趋近于零, 分别为 $0 \pm 0.39 \%$ 和 $0 \pm 0.72 \%$. 来自现代洋中脊热液区 的硫化物的 $\delta^{82 / 76} \mathrm{Se}_{\mathrm{NIST}}$ 为 $(-1.26 \pm 2.6) \%{ }^{[9]}$. 现代海底 沉积物的 $\delta^{82 / 76} \mathrm{Se}_{\mathrm{NIST}}$ 为 $(-0.53 \pm 2.0) \% \mathrm{o}^{[10]}$. Hagiwara ${ }^{[11]}$ 测定了一系列黑色页岩的样品, 其中未风化和风化 (蚀变) 样品的 $\delta^{82 / 76} \mathrm{Se}_{\mathrm{NIST}}$ 分别为 $(1.23 \pm 1.00) \%$ 和 (0.76 \pm 3.30$) \%$. 图中也给出了中国其他地区黑色岩系 的 $\delta^{82 / 76} \mathrm{Se}_{\mathrm{NIST}}$ 值 (自测, 未发表数据), 其中遵义下寒 武统牛蹄塘组, 紫阳下寒武统鲁家坪组和塔里木下 寒武统玉尔吐斯组黑色岩系的 $\delta^{82 / 76} \mathrm{Se}_{\mathrm{NIST}}$ 变化于 $-1.50 \%$ 1.85\%o, 平均 $(0.48 \pm 1.04) \%$. 总体上, 沉积 岩中的硒同位素趋向于富集重同位素. 拉尔玛地区 下寒武统太阳顶群地层显示了较大的同位素分馏, 并且富集轻同位素, 其 $\delta^{82 / 76} \mathrm{Se}_{\mathrm{NIST}}$ 变化于 $-2.44 \%$ $-9.35 \%$, 平均 $(-4.80 \pm 2.92) \%$. 因此, 对比上述所有 目前得到的测试数据, 渔塘坝硒矿床的硒同位素测 试数据显示了最大的同位素分馏和最大的轻同位素 富集.

研究已经证实硒同位素的主要分馏作用是硒酸 盐和亚硒酸盐的无机和微生物(细菌)还原过程造成 的. Johnson ${ }^{[12]}$ 综合目前的模拟实验, 认为从 $\mathrm{Se}(\mathrm{VI})$

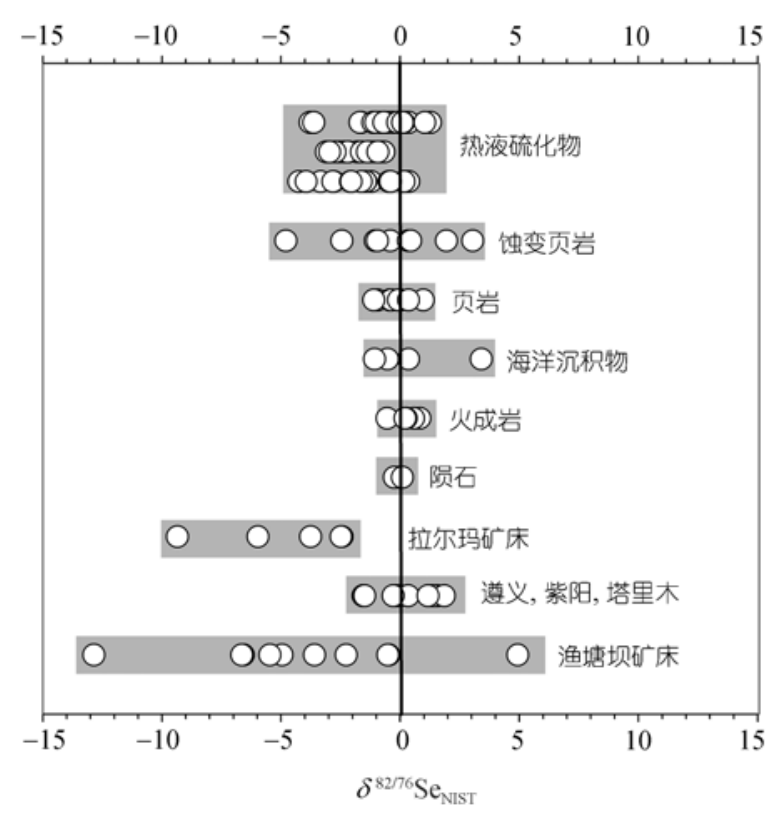

图 1 自然地质样品硒同位素分布

到Se(IV)和 Se(IV) 到Se(0)的无机还原分别能造成 10\%。 18\%o和 9\%o 18\%o的同位素分馏, 在微生物还原 作用下的同位素分馏稍小, 分别为 $4 \%$ \% 7\%o和 $9 \%$ 14\%. 因此, 海水中的高价态硒在缺氧条件下通过无 机或微生物还原成自然硒显然应该有较大的同位素 分馏. 然而一些证据显示: ( i ) 海水中硒的含量是很 低的(约 $2 \mathrm{nmol} / \mathrm{L}$ ), 通过无机还原的方式要累积如此 高的硒含量是极端困难的 ${ }^{[13]}$. (ii) Cutter ${ }^{[14]}$ 对海水柱 的测定表明, 从表层氧化条件到底层还原环境, 高价 态硒(硒酸盐和亚硒酸盐)急剧减少, 几乎完全转换为 有机态硒, 而有机态硒则主要与浮游植物的吸收有关; 同时, Baines 等人 ${ }^{[15]}$ 的研究表明, 只有通过藻类和

表 1 渔塘坝硒矿床中硒同位素组成

\begin{tabular}{|c|c|c|c|c|c|c|c|}
\hline 样号 & 岩性 & $\mathrm{Se} / \mu \mathrm{g} \cdot \mathrm{g}^{-1}$ & $\mathrm{~S} / \%$ & Corg/\% & $\mathrm{C} / \mathrm{N}$ & $\delta^{82 / 76} \mathrm{Se}_{\mathrm{NIST}} / \%$ & $2 \sigma$ \\
\hline Ytb-1 & 含碳硅质岩 & 3470 & 0.53 & 9.89 & 40 & 4.93 & 0.21 \\
\hline Ytb-2 & 碳质页岩 & 4646 & 1.48 & 27.74 & 33 & -3.60 & 0.02 \\
\hline Ytb-3 & 碳质页岩 & 191 & 0.4 & 19.26 & 35 & -4.83 & 0.25 \\
\hline Ytb-4 & 含碳硅质岩 & 2080 & 0.43 & 9.7 & 42 & -4.96 & 0.75 \\
\hline Ytb-5 & 碳质页岩 & 3721 & 1.56 & 33.28 & 43 & -12.77 & 0.15 \\
\hline Ytb-6 & 碳质页岩 & 1754 & 0.62 & 29.04 & 23 & -0.47 & 0.82 \\
\hline Ytb-8 & 碳质页岩 & 28 & 0.5 & 25.55 & 21 & -6.58 & 0.24 \\
\hline Ytb-12 & 含碳硅质岩 & 2546 & 0.49 & 11.99 & 43 & -6.93 & 0.10 \\
\hline $\mathrm{T} 2$ & 土壤 & 14.2 & 0.02 & 1.14 & 6 & -2.27 & 0.20 \\
\hline $\mathrm{T} 4$ & 土壤 & 6.7 & 0.03 & 1.12 & 5 & -0.52 & 0.20 \\
\hline
\end{tabular}


浮游植物的吸收才可能累积大于海水中几个数量级 的硒. 根据Hagiwara ${ }^{[11]}$ 的藻类吸收模拟实验和Herbel 等人 ${ }^{[16]}$ 的实际测量, 浮游植物(藻类等)或高等植物的 吸收不会造成大的硒同位素分馏, 一般在 1\% 2\%o. (iii) 根据我们收集的和自测的大部分黑色岩系样品, 硒同位素分布范围一般较窄, 其 ${ }^{82 / 76} \mathrm{Se}_{\mathrm{NIST}}$ 值一般变 化于-2\%o +2\% 之间, 并且趋向于略富集重同位素, 这一系列新鲜黑色页岩样品中硒同位素测试数据的 分布显然不支持这一机制. 值得注意的是, 在沉积岩 中, 硫同位素往往有比较宽的分布范围, 其 $\delta^{34} \mathrm{~S}$ 变化 范围可达 $150 \%{ }^{[17]}$. Johnson和Bullen ${ }^{[18]}$ 推测硒与硫在 海水中的生物地球化学循环截然不同, 硫一般由海 水中的硫酸盐通过无机或微生物作用还原从而造成 了较大的同位素分馏. 硒则主要通过浮游植物的吸 收而沉积; (iv) 理论上, 细菌和微生物的异化作用能 还原高价态的海水中的(亚)硒酸盐到单质硒 $\left(\mathrm{Se}^{0}\right)$ 和 硒化氢 $\left(\mathrm{H}_{2} \mathrm{Se}\right)$, 然而, Zehr和Oremland ${ }^{[19]}$ 认为在实际 地质条件下, 这一过程并不是硒的生物地球化学循 环的主要形式. 硫化物中的硒可能是有机结合态的 硒在有机质降解过程中释放出部分游离的 -2 价硒而 通过类质同象替换了硫化物中部分的硫. 这一过程 也不会造成大的硒同位素分馏, 即使部分游离的 -2 价硒被氧化到 0 价态硒, 其同位素分馏也是很小的 ${ }^{[17]}$. (v) 尽管目前尚无海水的硒同位素数据, 然而根据 现代海底锰结核 (大致代表海水平均值)的测试, 其 $\delta^{82 / 76} \mathrm{Se}_{\mathrm{NIST}}$ 值约为 $0.35 \%$ ，表明海水与沉积物之间并 没有明显的硒同位素分馏 [8]. 综合以上, 我们认为海 水中的高价态硒在缺氧条件下通过无机或微生物还 原成自然硒这一模式的可能性是非常小的. 值得注 意的是, 拉尔玛下寒武统太阳顶群地层中也具有较 大的同位素分馏, 然而我们前期研究表明其大约

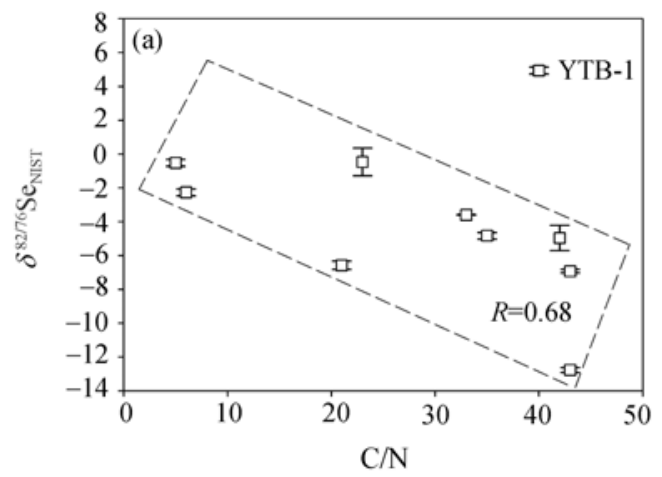

$75 \%$ 的硒以有机结合态的形式存在, 因此它的较大的 同位素分馏可能联系到成岩期的微生物的异化作用, 早期的浅变质作用和后期的热液成矿活动 (另文讨 论), 而与渔塘坝硒矿床中单质硒的大量出现在机制 和成矿演化上是完全不同的 ${ }^{[2,3,20]}$. 例如, 在渔塘坝硒 矿床中并没有后期热液叠加的痕迹. 此外, “石煤”的 自燃或构造热的出融作用实际上是一个快速氧化的 过程, 实验已经证明硒的氧化不会造成大的硒同位 素分馏 ${ }^{121}$.

正如在我们先前的研究所指出的, 硒的富集机 制可能与地下水的氧化淋滤次生富集过程密切相 关 ${ }^{[3,4]}$. 勘探结果已经表明, 渔塘坝硒矿床中全部矿 体均位于现代潜水面以上, 并且从地表到潜水面下, 硒含量呈低(上部氧化带)一高(下部氧化带)一低(原 生带)的分布模式 ${ }^{[4]}$. 尽管目前尚无矿床形成的年龄 数据, 然而, 我们倾向于认为这一过程是动态的, 随 着潜水面的上下波动, 硒也随之不断重新分布. 因此, 根据实际的勘探结果, “氧化-还原模式”可能能较好 的解释存在的硒同位素分馏. 当原先赋存于原岩中 的低价态硒(有机结合态和硫化物结合态)在潜水面 上下被富含氧的地下水氧化到高价态的硒时, 按照 目前的实验结果, 氧化作用并不会发生明显的同位 素分馏 ${ }^{[11]}$. 然而当高价态的含硒溶液在向下渗滤的 过程中, 由于氧化还原条件的改变, 特别是碳质的强 还原作用, 使得高价态硒重新被还原, 将硒重新还原 成 0 价(单质Se), 部分被还原成 -2 价呈硒化物或类质 同象形式存在, 但其形成的条件苛刻的多(已越过水 的稳定下限). 在这一过程中, 以无机还原为主, 硒同 位素可发生较大的同位素分馏.

除地质证据外，硒同位素与 $\mathrm{C} / \mathrm{N}$ 比值的相关关 系也部分的支持这一推断(图 2(a)). 通常的, C/N 比值

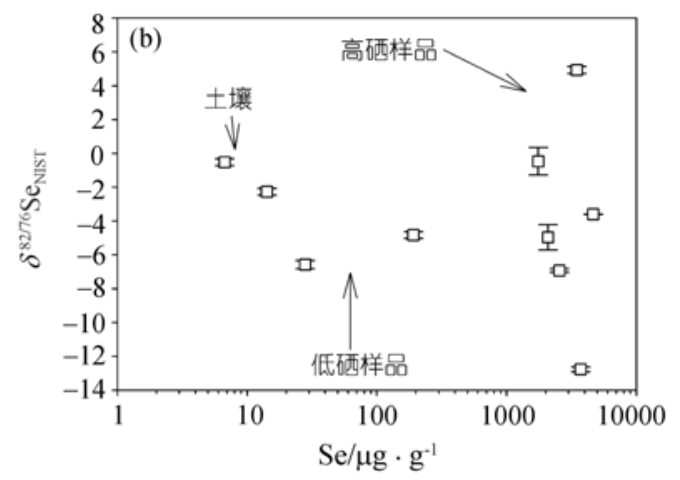

图 2 硒同位素组成与 C/N 比值关系. 相关系数计算去除了样品 YTB-1 (a), 硒同位素组成与硒含量的关系(b) 
可近似代表岩石受蚀变的强度, 因为在有机质蚀变 过程中, $\mathrm{N}$ 的丢失要快于 $\mathrm{C}$ 的丢失 ${ }^{[21]}$. 因此, 这意味着 $\mathrm{C} / \mathrm{N}$ 比值越高的样品, 则遭受氧化还原作用越强, 部 分的, 可能也暗示在这些高 $\mathrm{C} / \mathrm{N}$ 比值的样品中, 氧化 还原作用可能多次进行，这一机制必然导致更大的 硒同位素分馏. 图 2(b)中, 低硒样品则保持相对一致 的分布, 富集轻同位素, 而高硒样品中硒同位素显示 了一个较大的分布范围, 从 $+4.93 \%$ - $12.77 \%$, 特别 是所测样品中一个较大的 $\delta^{82 / 76} \mathrm{Se}_{\mathrm{NIST}}$ 正异常 (Ytb-1)的 发现, 可能暗示了同位素动力分馏的存在.

正如Johnson ${ }^{[12]}$ 所指出的, 由 0 价硒(自然硒)还原 到-2 价硒(硒化物或硫化物类质同象)这一过程中并 没有同位素分馏的存在. 因此，我们用全岩样品的硒 同位素组成去解释渔塘坝硒矿床中硒的富集机制, 特别是其中自然硒的形成机制，一定意义上，全岩样 品的硒同位素组成可近似代表干酪根中自然硒的硒 同位素组成. 当然，更完善的模式，尚需更多的测试 工作去构建. 一些样品的解释也需要其他证据的支 持, 如土壤样品的解释. 然而, 用硒同位素证据来阐 明渔塘坝硒矿床中硒的地球化学循环及其富集机制, 部分证明了“氧化-还原模式”是解释矿床中自然硒形 成的合理模式. 同时，在矿床中所发现的目前自然界 中最大的硒同位素分馏和最大的轻同位素富集也间 接论证了硒同位素作为一种新的地球化学示踪剂有 其独特的应用潜力.

致谢 感谢法国 CRPG-CNRS 实验室的 Luc Marin 和 Céline Fournier 以及法国里昂 ENS-Lyon 实验室的 P. Telouk 提供的技术支持.

\section{参考文献}

1 朱建明, 郑宝山, 刘世荣, 等. 多形态自然硒的首次发现及其成 因初探. 矿物学报, 2000, 20(4): 337-341

2 温汉捷, 䒾愉卓, 刘世荣. 硒在干酪根中的两种不同赋存状态 TEM 证据. 地球化学, 2003, 32(1): 21-28

3 Wen H J, Carignan J, Qiu Y Z, et al. Selenium Speciation in Kerogen from Two Chinese Selenium Deposits: Environmental Implications. Environ Sci Techol, 2006, 40: 1126-1132[DOI]

4 王鸿发, 李均权. 湖北恩施双河硒矿矿床地质特征. 湖北地质, 1996, 10(2): 10-21

5 姚林波, 高振敏, 杨竹森, 等. 渔塘坝硒矿床富硒硅质岩的成因.
中国科学 D 辑: 地球科学, 2002, 32(1): 54-63

6 朱建明, 郑宝山, 苏宏灿, 等. 恩施渔塘坝自然硒的发现及其初 步研究. 地球化学, 2001, 30(3): 236-241

7 Marin L, Lhomme J, Carignan J. GFAAS determination of selenium after separation with thiol cotton in lichens and plants: The importance of adding a mineral matrix before decomposition. Talanta, 2003, 61(2): 119-125 [DOI]

8 Carignan J, Wen H J. Scaling NIST SRM 3149 for Se isotope analysis and isotopic variations of natural samples. Chem Geol, 2007, doi: 10.1016/j.chemgeo.2007.03.020

9 Rouxel O, Ludden J, Carignan J, et al. Natural variations of Se isotopic composition determined by hydride generation multiple collector inductively coupled plasma mass spectrometry. Geochim Cosmochim Acta, 2002, 66(18): 3191-3199[DOI]

10 Rouxel O, Fouquet Y, Ludden J. Subsurface processes at the lucky strike hydrothermal field, Mid-Atlantic ridge: Evidence from sulfur, selenium, and iron isotopes. Geochim Cosmochim Acta, 2004, 68(10): 2295-2311 [DOI]

11 Hagiwara Y. Selenium isotope ratios in marine sediments and algae: A reconnaissance study. Dissertation for the Master Degree. Urbana, IL: Univ of Illinois at Urbana-Champaign, 2000

12 Johnson T M. A review of mass-dependent fractionation of selenium isotopes and implications for other heavy stable isotopes. Chem Geol, 2004, 204(3-4): 201-214 [DOI]s

13 Cutter G A, Bruland K W. The marine biogeochemistry of selenium: A re-evaluation. Limnol Oceanogr, 1984, 29(6): 1179—1192

14 Cutter G A. Selenium in Reducing Waters. Science 27 August, 1982, 217: 829-831

15 Baines S B, Fisher N S, Doblin M A, et al. Uptake of dissolved organic selenides by marine phytoplankton. Limnol Oceanogr, 2001, 46, 1936-1944

16 Herbel M J, Johnson T M, Tanji K K, et al. Selenium stable isotope ratios in California agricultural drainage water management systems. J Environ Qual, 2002, 31(July-August): 1146-1156

17 郑永飞, 陈江峰. 稳定同位素地球化学. 北京: 科学出版社, 2000. $1-316$

18 Johnson T M, Bullen T D. Mass-dependent fractionation of selenium and chromium isotopes in low-temperature environments, In: Rosso J J, ed. Geochemistry of Non-traditional Stable Isotopes. Washington DC: The Mineralogical Society of America, 2003. 289-317

19 Zehr J P, Oremland R S. Reduction of selenate to selenide by sulfate-respiring bacteria: Experiments with cell suspensions and estuarine sediments. Appl Environ Microbiol, 1987, 53: 1365—1369

20 Wen H J, Qiu Y Z. Geology and Geochemistry of Se-Bearing Formations in Central China. Int Geol Rev, 2002, 44(2): 164-178.

21 傅家谟, 秦匡宗. 干酪根地球化学. 广州: 广东科技出版社. 1995. 637 